\title{
Pacemaker lead extraction and recapture of venous access: Technical problems arising from extensive venous obstruction
}

\author{
Andrzej Kutarski ${ }^{1}$, Radosław Pietura ${ }^{2}$, Krzysztof Młynarczyk ${ }^{3}$, \\ Barbara Małecka ${ }^{4}$, Andrzej Głowniak ${ }^{1}$ \\ ${ }^{1}$ Department of Cardiology, Medical University of Lublin, Poland \\ ${ }^{2}$ Department of Interventional Radiology and Neuroradiology, Medical University of Lublin, Poland \\ ${ }^{3}$ Department of Cardiology, Specialistic Hospital, Tarnow, Poland \\ ${ }^{4}$ Department of Electrocardiology, John Paul II Hospital, Krakow, Poland
}

\begin{abstract}
We report the case of the extraction of 18 year-old leads in a patient with a DDD pacemaker, and chronic obstruction of the left subclavian and innominate veins coexisting with extensive stenoses in the upper caval vein. After removal of pacing leads, angiographic guidewires were introduced via the Byrd dilatators and new pacing leads introduced with the use of long sheaths originally dedicated for transvenous left ventricular leads implantation. With this case, we discuss the problems arising during reimplantation of pacing leads in patients with chronic venous occlusion. (Cardiol J 2012; 19, 5: 513-517)
\end{abstract}

Key words: lead-related venous occlusion, lead extraction, recapture of venous access

\section{Introduction}

Occlusion or significant stenosis of great chest veins are common consequences of permanent transvenous pacing, occurring in $15-30 \%$ of patients implanted with a cardiac pacemaker [1-4]. Repetitive, chronic friction between the pacing lead and the endothelium can initiate the inflammatory process, leading to thrombosis and fibrosis, resulting in separation of the pacing lead from the bloodstream. This leads to compensatory dilatation or, on the contrary, stenosis/occlusion of the vein [4-8]. This occlusion, usually symptomless due to the development of by-pass circulation, can cause major trouble during lead extraction [1-4].

Current guidelines do not recommend the use of venous access from the opposite side during inplantation of the new pacing leads (which should remain patent for other medical purposes, like dialysis catheters or central venous line). Instead, recapture of the venous approach from the same side is recommended [9], with the use of dedicated tools, of which the sheaths dissecting the extracted lead from the surrounding tissues are most commonly used. After the lead is removed, the sheath allows the introduction of the guidewires (and subsequently new pacing leads) to the heart. In less extensive cases, reimplantation of the pacing lead with the standard introducer is possible [10]. However, more and more frequently, the extensive obstruction of the venous system forces us to reach for non-standard techniques. We report a case illustrating this aspect of the 'dark side' of electrotherapy.

\section{Case report}

An 83 year-old woman, implanted with a DDD pacemaker 18 years before (pacing leads in right atrial appendage and right ventricular apex), was referred to our center due to malfunction of both

Address for correspondence: Andrzej Kutarski, MD, PhD, Department of Cardiology, Medical University of Lublin, ul. Jaczewskiego 8, 20-954 Lublin, Poland, tel/fax: +48 81724 41 51, e-mail: a_kutarski@yahoo.com 


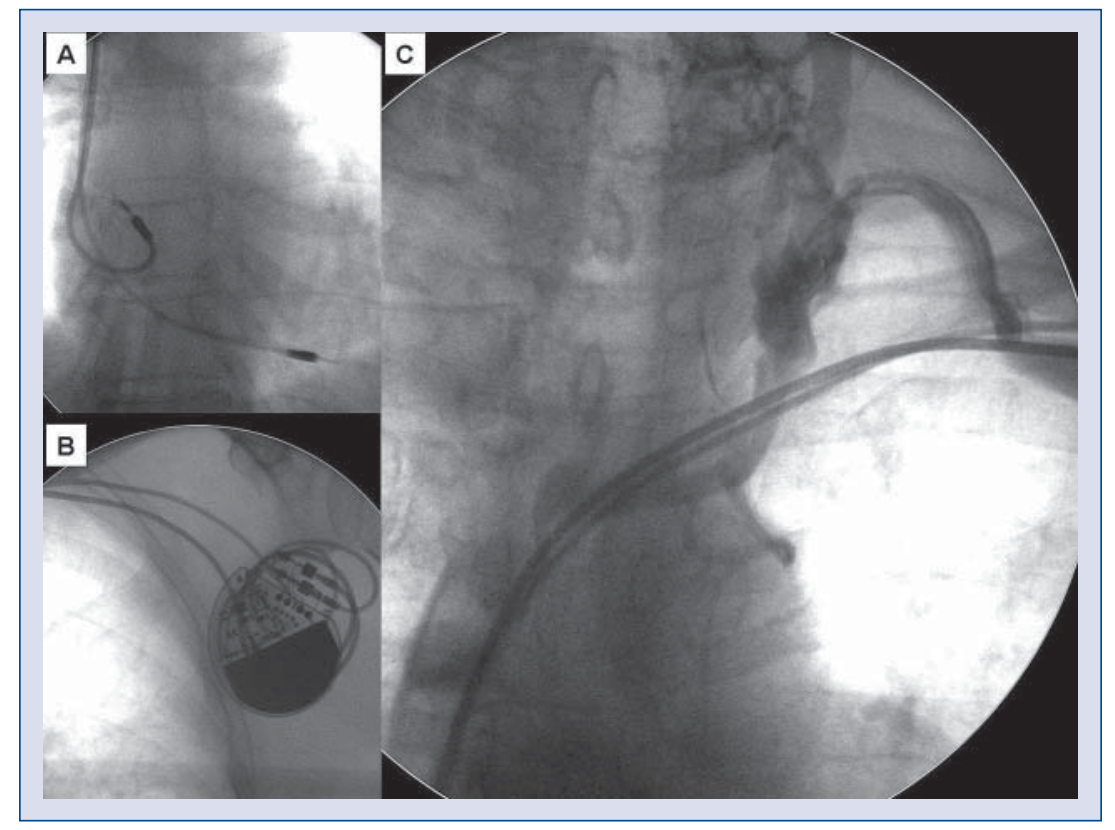

Figure 1. Chest X-ray (A, B) and baseline venography (C).

leads (Medtronic company, bipolar, passive, polyurethane external coating). During the last pacemaker re-implantation (two years before), the leads were reported to have low impedance and increased pacing threshold. However, they were not replaced due to diagnosed venous occlusion. On admission, both leads had impedance $<200 \Omega$ in both uni- and bipolar configuration, pacing threshold $6.0 \mathrm{~V} / 0.5 \mathrm{~ms}$ on ventricular lead, exit block on atrial lead and not acceptable signals on both leads. The patient was in good overall status with laboratory findings within normal limits. Echocardiographic examination (otherwise normal), revealed a $1.3 \mathrm{~cm}$ long highly mobile structure in the right atrium (RA), most likely a thrombus.

Fluoroscopic examination confirmed normal position and course of the pacing leads, while venography revealed occlusion of subclavian and innominate (brachiocephalic) veins and irregular contrast filling in upper caval vein (Fig. 1). After stable junctional rhythm of $50 \mathrm{bpm}$ was confirmed, we opened the pacemaker pocket, cut the ligatures, and began the lead extraction procedure.

We started with the atrial lead, which was extracted unbroken, despite a tight connective tissue sleeve (Figs. 2A-C). The normally straightforward introduction of the angiographic guidewire into the RA met obstacles in the superior vena cava (SVC), although after several attempts this was successful (Figs. 2D, E). Next, the ventricular lead was extracted in the same way, again with obstacles when passing the guidewire through the SVC (Figs. 2F, G).

A standard introducer was passed over the guidewire behind the occlusion. Contrast injection revealed a double tunnel cut by Byrd dilatators, ending at the level of high RA with no further contrast flow (Figs. 3A-C). We decided to use long introducers dedicated for the coronary sinus (Medtronic Attain, $7 \mathrm{~F}$ diameter), allowing the introduction of thin (Biotronic Setrox) pacing leads (Figs. 3D, E; 4A-C). Since the introducer sheath was longer than the pacing lead, we had to simultaneously cut along the sheath (using a standard cutting instrument) during the introduction of the pacing lead.

This maneuver (which we had performed several times previously) was successful, and we avoided unintentional damage to the leads by the cutting tool. The subsequent course of the procedure was uncomplicated. Figure 4D presents the extracted lead, with no visible signs of damage. The possible cause of leads malfunction with slow impedance decrease could be a gradual damage to the inner insulation separating the coils.

Control pre-discharge angiography (with DSA mode) was performed, revealing extensive remodeling of venous flow secondary to the long-term (18 years) presence of pacing leads (Fig. 5). The axillary, subclavian, brachicephalic and upper caval veins on the right side were patent. On the left side, only the axillary, and the distal part of the subclavian, vein remained patent. The proximal part of the 
Andrzej Kutarski et al., Recapture of venous access in extensive vein obstruction

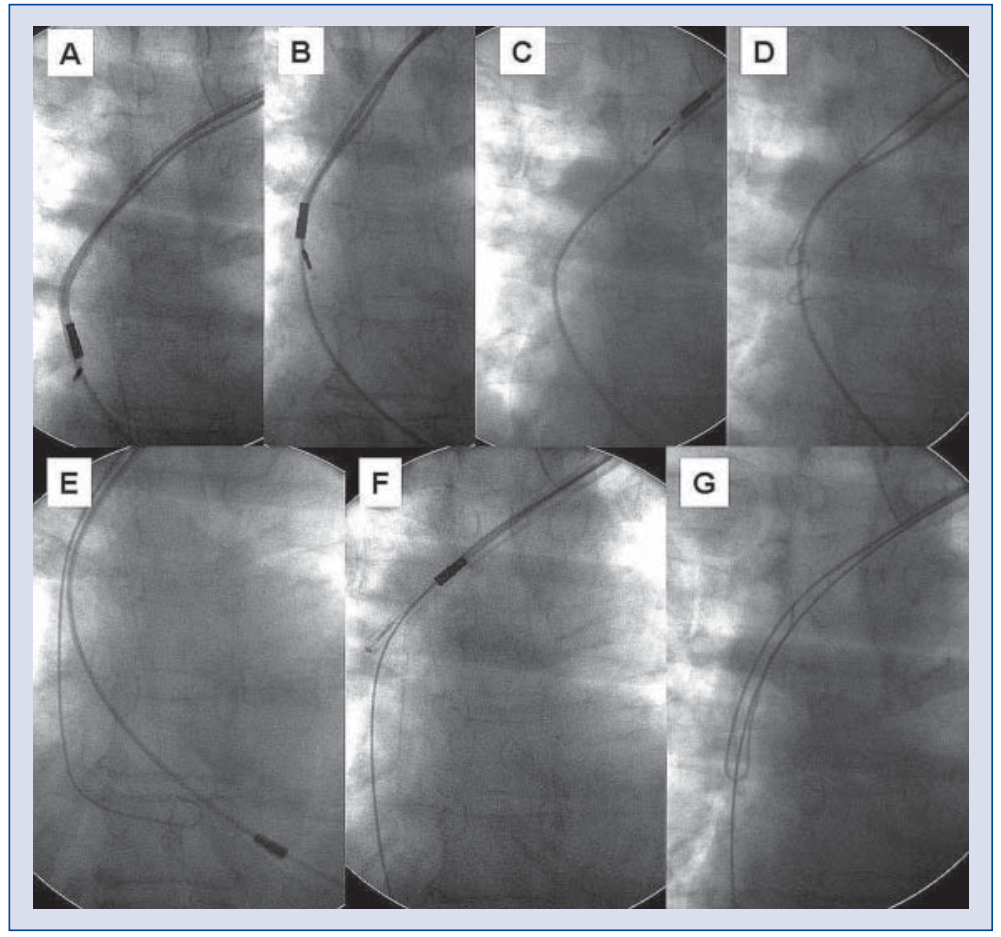

Figure 2. Subsequent stages of lead extraction.

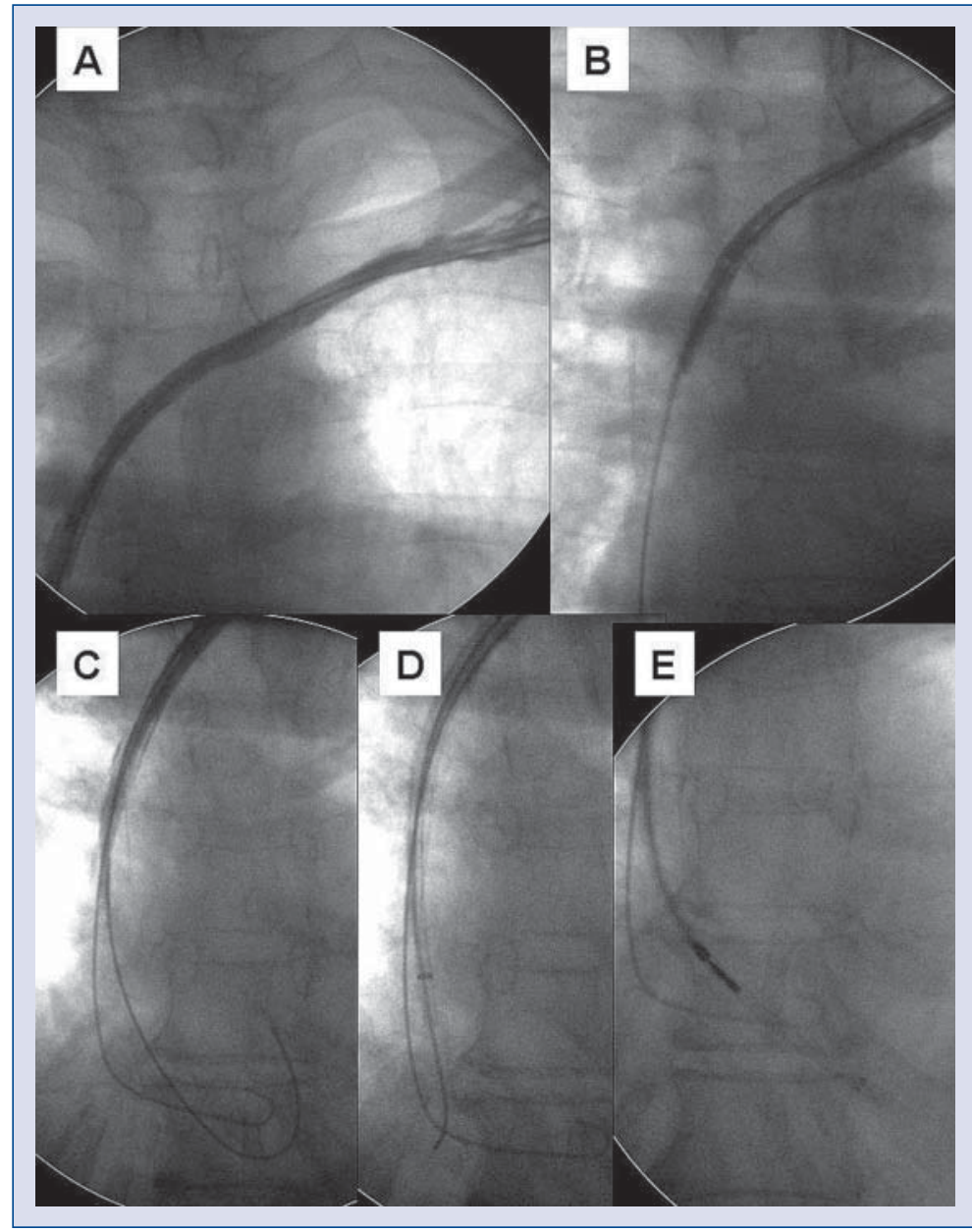

Figure 3. Implantation of the new pacing leads - step 1. 
Cardiology Journal 2012, Vol. 19, No. 5

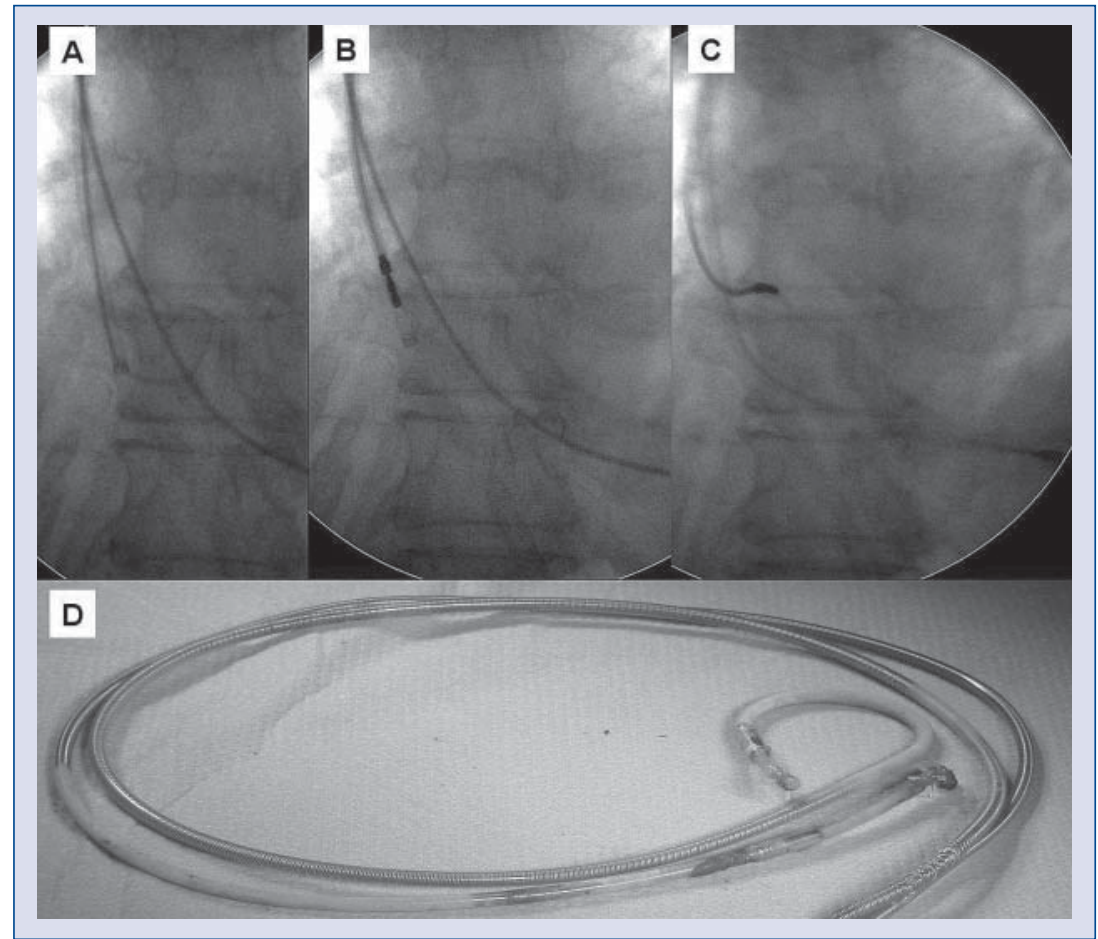

Figure 4. Implantation of the new pacing leads - step 2.

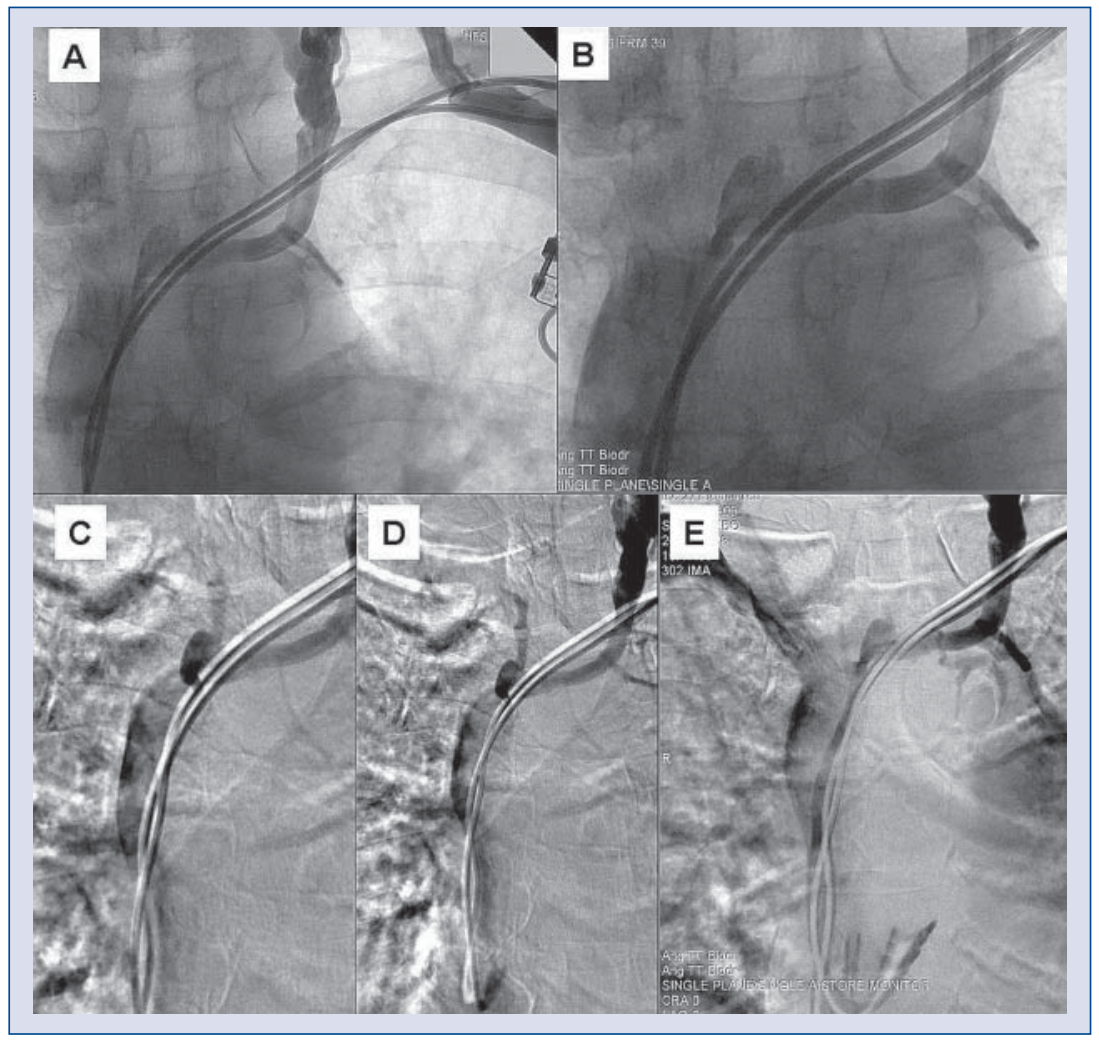

Figure 5. Control venography after the procedure. 
left subclavian vein was occluded with huge by-pass flow through the dilated jugular veins. The picture of the left brachiocephalic vein was unclear, with a by-pass vessel $5 \mathrm{~mm}$ in diameter parallel to the leads. Moreover, both extracted pacing leads, during their course through SVC, were surrounded by a sleeve, separating them from the SVC lumen - new leads were introduced via the same access (there was obviously no direct entry to the main lumen of SVC from the left sided veins). Additionally, mild SVC stenosis just above the entrance to the RA was confirmed. However, all these changes in the venous flow were asymptomatic and the patient was discharged home in good overall status.

\section{Discussion}

The described case illustrates difficulties we often come across during extraction of old, malfunctioning pacing leads. Multilevel venous stenoses/ /obstruction and fake 'extravascular' course of the leads are typical findings. In our patient, obstruction of the venous flow was extensive, making the extraction procedure more complicated. It demonstrates the need for non-standard, dedicated tools, like introducers with a long, peel-away sheath. This will help to avoid possible damage to the leads while cutting away the sheath, originally designed for other purposes.

There are obviously some alternative approaches to this problem. Firstly, it is possible to pass the angiographic guidewire through the stenotic area with the use of a cutting (laser, electrocautery or mechanical) sheath, and, subsequently dilate the occlusion with a balloon. Such balloon angioplasty usually provides only a transitory effect, yet sufficient to introduce the new pacing leads $[11,12]$. Although venoplasty allows the addition/ /replacement of leads in the case of subtotal or complete venous occlusion, it is rarely performed because many implanting physicians are not familiar with the balloon technique.

Alternatively, when it is possible to pull the extracted lead through the stenotic area, a long guidewire inserted inside the lead insulation can be pulled down along with the lead to the femoral vein. Then, the lead is extracted via the femoral access, and the new lead is implanted with the use of the guidewire via the subclavian vein $[13,14]$. If the inactive lead should be left in place (e.g. for safety reasons), the new lead can be implanted after the venous access is recaptured with the use of a laser sheath inserted over the inactive lead [15]. It is also possible to pass the new lead through the Byrd dila- tor, which is subsequently cut away with the instrument dedicated for coronary sinus sheaths (frequently used in our lab, although unpublished technique).

The described case along with the discussed literature confirm the truism that even an inactive, damaged lead can be an excellent key to recapture the access to the heart in a case of lead-dependent venous occlusion.

\section{Conflict of interest: none declared}

\section{References}

1. Bracke FA, Meijer A, Van Gelder LM. Symptomatic occlusion of the access vein after pacemaker or ICD lead extraction. Heart, 2003; 89: 1348-1349.

2. Bracke F, Meijer A, Van Gelder B. Venous occlusion of the access vein in patients referred for lead extraction: Influence of patient and lead characteristics. Pacing Clin Electrophysiol, 2003; 26: 1649-1652.

3. Korkeila P, Nyman K, Ylitalo A et al. Venous obstruction after pacemaker implantation. Pacing Clin Electrophysiol, 2007; 30: 199-206.

4. Lickfett L, Bitzen A, Arepally A et al. Incidence of venous obstruction following insertion of an implantable cardioverter defibrillator. A study of systematic contrast venography on patients presenting for their first elective ICD generator replacement. Europace, 2004; 6: 25-31.

5. Jones GK, Swerdlow C, Reichenbach DD et al. Anatomical findings in patients having had a chronically indwelling coronary sinus defibrillation lead. Pacing Clin Electrophysiol, 1995; 18: 2062-2067.

6. Esposito M, Kennergren C, Holmström N et al. Morphologic and immunohistochemical observations of tissues surrounding retrieved transvenous pacemaker leads. J Biomed Mater Res, 2002; 63: 548-558.

7. Forauer AR, Theoharis C. Histologic changes in the human vein wall adjacent to indwelling central venous catheters. J Vasc Interv Radiol, 2003; 14: 1163-1168.

8. Candinas R, Duru F, Schneider J et al. Postmortem analysis of encapsulation around long-term ventricular endocardial pacing leads. Mayo Clin Proc, 1999; 74: 120-125.

9. Wilkoff BL, Love CJ, Byrd CL et al. Heart Rhythm Society; American Heart Association. Transvenous lead extraction: Heart Rhythm Society expert consensus on facilities, training, indications, and patient management. This document was endorsed by the American Heart Association (AHA). Heart Rhythm, 2009; 6: 1085-1104.

10. Henrikson CA, Alexander D, Ringel RE et al. Laser recanalization of the subclavian vein. Pacing Clin Electrophysiol, 2006; 29: 436-437.

11. Worley SJ. Implant venoplasty: Dilation of subclavian and coronary veins to facilitate device implantation: Indications, frequency, methods, and complications. J Cardiovasc Electrophysiol, 2008; 19: 1004-1007.

12. Pace JN, Maquilan M, Hessen SE et al. Extraction and replacement of permanent pacemaker leads through occluded vessels: use of extraction sheaths as conduits: Balloon venoplasty as an adjunct. J Interv Card Electrophysiol, 1997; 1: 271-279.

13. Staniforth AD, Schilling RJ. Reuse of occluded veins during permanent pacemaker lead extraction: A new indication for femoral lead extraction. Indian Pacing Electrophysiol J, 2002; 2: 97-103.

14. Rogers DP, Lambiase PD, Chow AW. Successful coronary sinus lead replacement despite total venous occlusion using femoral pull through, two operator counter-traction and subclavian venoplasty. J Interv Card Electrophysiol, 2007; 19: 69-71.

15. Bracke FA, Meijer A, van Gelder LM. Use of a laser sheath to obtain venous access in pacemaker lead-related obstruction without extraction of the lead. Europace, 2002; 4: 67-68. 